\title{
Combination of Morphologic Criteria and $\alpha$-Fetoprotein in Selection of Patients With Hepatocellular Carcinoma for Liver Transplantation Minimizes the Problem of Posttransplant Tumor Recurrence
}

\author{
Michał Grąt • Oskar Kornasiewicz • Zbigniew Lewandowski • Wacław Hołówko • \\ Karolina Grąt • Konrad Kobryń • Waldemar Patkowski • Krzysztof Zieniewicz • \\ Marek Krawczyk
}

Published online: 24 May 2014

(C) The Author(s) 2014. This article is published with open access at Springerlink.com

\begin{abstract}
Background Serum $\alpha$-fetoprotein concentration (AFP) might be a useful addition to morphologic criteria for selecting patients with hepatocellular carcinoma (HCC) for liver transplantation (LT). The aim of this study was to evaluate the role of AFP in selecting HCC patients at minimal risk of posttransplant tumor recurrence in the setting of existing criteria.

Methods This retrospective cohort study was based on 121 HCC patients after LT performed at a single institution. AFP was evaluated as a predictor of posttransplant tumor recurrence with respect to fulfillment of the Milan, University of California, San Francisco (UCSF), and Upto-7 criteria.

Results There was a nearly linear association between AFP and the risk of HCC recurrence $(p<0.001$ for linear effect; $p=0.434$ for nonlinear effect). AFP predicted HCC recurrence in patients (1) beyond the Milan criteria $(p<0.001$; optimal cutoff $200 \mathrm{ng} / \mathrm{ml})$; (2) within the UCSF criteria $(p=0.001$; optimal cutoff $100 \mathrm{ng} / \mathrm{ml})$ and beyond them $(p=0.015$; optimal cutoff $200 \mathrm{ng} / \mathrm{ml})$; and (3) within the Up-to-7 criteria $(p=0.001$; optimal cutoff $100 \mathrm{ng} / \mathrm{ml})$ and beyond them $(p=0.023$; optimal cutoff $100 \mathrm{ng} / \mathrm{ml}$ ) but not in patients within the Milan criteria $(p=0.834)$. Patients within either UCSF and
\end{abstract}

M. Grąt $(\bowtie) \cdot$ O. Kornasiewicz · W. Hołówko · K. Grąt ·

K. Kobryń · W. Patkowski - K. Zieniewicz · M. Krawczyk

Department of General, Transplant and Liver Surgery, Medical

University of Warsaw, 1A Banacha Street, 02-097 Warsaw,

Poland

e-mail: michal.grat@gmail.com

Z. Lewandowski

Department of Epidemiology, Medical University of Warsaw, 3

Oczki Street, 02-007 Warsaw, Poland
Up-to-7 criteria with AFP level $<100 \mathrm{ng} / \mathrm{ml}$ exhibited superior $(100 \%) 5$-year recurrence-free survival-significantly higher than those within UCSF $(p=0.005)$ or Upto-7 ( $p=0.001)$ criteria with AFP levels higher than the estimated cutoffs or beyond with AFP levels less than the estimated cutoffs.

Conclusions Combining the UCSF and Up-to-7 criteria with an AFP level $<100 \mathrm{ng} / \mathrm{ml}$ is associated with minimal risk of tumor recurrence. Hence, this combination might be useful for selecting HCC patients for LT.

\section{Introduction}

Hepatocellular cancer (HCC) is the most frequent of the primary malignancies of the liver, ranked second and sixth on the list of the most common causes of tumor-related mortality worldwide in men and women, respectively [1]. Considering the unfavorable prognoses of untreated patients and only moderate response to chemotherapy [2, 3], surgical treatment is of the utmost importance. Unfortunately, given that the majority of these tumors occur in cirrhotic livers, the use of liver resection is often limited by the presence of portal hypertension and poor hepatic function, as reflected by the guidelines of the Barcelona Clinic Liver Cancer Group [4]. In contrast, liver transplantation (LT) not only removes all macroscopic and microscopic hepatic tumors with almost no risk of positive oncologic margins, it cures the underlying cirrhosis.

Early experiences with LT for HCC were characterized by an unacceptably high risk of posttransplant tumor recurrence and its related high mortality rate [5]. This situation was due to a lack of specific selection criteria and performing LT often in patients with large and multifocal tumors. A new era began with adoption of the Milan 
criteria into clinical practice (single tumor $\leq 5 \mathrm{~cm}$ or two or three tumors $\leq 3 \mathrm{~cm}$, with no evidence of extrahepatic metastases or macrovascular invasion) [6]. Based on the results of several observational studies, expansions of these restrictive criteria have been proposed. One criteria set is that of the University of California, San Francisco (UCSF) with criteria of a single tumor $<6.5 \mathrm{~cm}$ or two or three tumors $<4.5 \mathrm{~cm}$, total tumor diameter $<8 \mathrm{~cm}$. Another criteria set is the Up-to-7 criteria (size of the largest tumor + number of tumors $\leq 7)[7,8]$. Even though the conservative Milan criteria are still widely used for selecting patients with HCC to undergo LT, outcomes after LT for HCC remain inferior to those performed for benign indications [9, 10]. Because the inferior outcomes of $\mathrm{HCC}$ patients are mainly due to posttransplant tumor recurrence, the use of expansions of the Milan criteria that are already based solely on morphologic tumor characteristics would not eliminate these discrepancies. During this era of severe shortage of available organs and increasing demand for LT, there is general agreement in the transplant community that posttransplant outcomes of HCC patients should be similar to those undergoing transplantation for other indications [11]. Optimization of the existing selection criteria seems crucial to achieve this goal.

Historically, the serum $\alpha$-fetoprotein (AFP) concentration was applied for HCC screening among high-risk groups and to establish the diagnosis. Considering its poor diagnostic features, it is no longer recommended in these clinical settings $[12,13]$. Nevertheless, AFP is currently gaining an increasing role as a marker of biologic aggressiveness of tumors. Numerous studies confirmed the prognostic significance of preoperative AFP in both liver resection and LT [14-18]. However, there is no agreement as to the most appropriate cutoff for AFP when selecting HCC patients for LT [11]. The purpose of this study was to evaluate the exact role of AFP in selecting HCC patients at minimal risk of posttransplant tumor recurrence in the setting of the existing criteria.

\section{Material and methods}

A total of 1,115 LTs were performed in the Department of General, Transplant, and Liver Surgery at the Medical University of Warsaw between December 1994 and June 2012. Basing on the results of explant pathology, $121 \mathrm{HCC}$ patients were included in this retrospective cohort study. Patients with combined hepatocellular/cholangiocellular cancer $(n=3)$, fibrolamellar HCC $(n=3)$, sarcomatous HCC $(n=1)$, or macroscopic vascular invasion $(n=6)$ were excluded. The ethics committee of the Medical University of Warsaw approved the study protocol. Details on the operative technique, postoperative immunosuppression, and follow-up visits were described previously [15, 19].
The last available pretransplant AFP was collected (usually measured within $24 \mathrm{~h}$ preceding transplantation). Tumor recurrences at 3 and 5 years were set as primary and secondary end points of the study, respectively. They were used to calculate the corresponding recurrence-free survival rates (observations were censored at the time of the last follow-up or death caused by conditions other than tumor recurrence). In general, risk factors for HCC recurrence at 3 years were established in univariate analyses. Subsequently, the associations between AFP and hazard of recurrence were adjusted for the impact of potential confounders in a series of bivariate analyses. Establishment of the optimal cutoffs for AFP for predicting $\mathrm{HCC}$ recurrence at 3 years was based on truepositive (TP) and false-positive (FP) prediction rates derived from estimated 3-year recurrence-free survival rates of patients with AFP levels higher and lower than the following values: $10,20,35,50$, and $100-1000 \mathrm{ng} / \mathrm{ml}$ (at $100 \mathrm{ng} / \mathrm{ml}$ intervals). TP and FP rates were calculated using the following equations:

$$
\begin{aligned}
& \mathrm{TP}=[a \times(1-\mathrm{RFSa})] /[(a \times(1-\mathrm{RFSa}))+(u \times(1-\mathrm{RFSu}))] \\
& \mathrm{FP}=(a \times \mathrm{RFSa}) /(a \times \mathrm{RFSa}+u \times \mathrm{RFSu})
\end{aligned}
$$

where $a$ is the number of patients with AFP above the analyzed value at risk at the start of the observation period; $u$ is the number of patients with AFP under the analyzed value at risk at the start of the observation period; RFSa is the estimated recurrence-free survival rate at 3 years for patients with AFP above the analyzed value; RFSu is the estimated recurrence-free survival rate at 3 years for patients with AFP under the analyzed value.

To the best of our knowledge, this novel methodologic approach allowed estimation of the optimal AFP cutoff values for predicting the 3-year recurrence rate even though the observation period was less than 3 years for some patients. For this reason, receiver operating characteristics curves were not used. Notably, the evaluation of the risk factors for HCC recurrence and AFP cutoffs was based on the first three posttransplant years when the vast majority of recurrent tumors are diagnosed [20]. Nevertheless, recurrence-free survivals of patients whose AFP levels were below and above the estimated AFP cutoffs were validated also within a time frame of five posttransplant years.

Data are presented as the median (range) for continuous variables and the number (\%) for categoric variables. Associations between AFP and categoric and continuous variables were assessed with the Mann-Whitney $U$ test and Spearman's correlation coefficient, respectively. Survival estimates were based on the Kaplan-Meier method. Survival curves were compared using a log-rank test. Cox's proportional hazards regression was used to evaluate risk 
Table 1 Characteristics of 121 patients included in the study cohort

Factor Median (range)

or no. (\%)

General factors
Recipient age (years)
Recipient sex
Female
Male
Child-Turcotte-Pugh class
A
B
C

Model for end-stage liver disease (points)

Hepatitis $C$ virus infection

Hepatitis B virus infection

Alcoholic cirrhosis

Donor age (years)

Donor sex

Female

Male

Cold ischemia (hours)

Fulfillment of selection criteria

\begin{tabular}{|c|c|}
\hline Milan & $67(55.4 \%)$ \\
\hline UCSF & $83(68.6 \%)$ \\
\hline Up-to-7 & $90(74.4 \%)$ \\
\hline \multicolumn{2}{|l|}{ Tumor-related factors } \\
\hline Serum AFP concentration (ng/ml) & $23.7(1.4-36208.0)$ \\
\hline Size of largest tumor $(\mathrm{cm})^{\mathrm{a}}$ & $3.4(0.3-14.0)$ \\
\hline No. of tumors ${ }^{\mathrm{a}}$ & $2(1-10)$ \\
\hline Total tumor volume $\left(\mathrm{cm}^{3}\right)^{\mathrm{a}, \mathrm{b}}$ & $22.5(0.02-5277.9)$ \\
\hline Microvascular invasion $^{\mathrm{a}}$ & $37(31.6 \%)$ \\
\hline Poor tumor differentiation ${ }^{\mathrm{a}}$ & $13(10.7 \%)$ \\
\hline Bilateral tumors ${ }^{\mathrm{a}}$ & $33(27.3 \%)$ \\
\hline Neodjuvant therapy & $42(34.7 \%)$ \\
\hline Chemoembolization & $22(18.2 \%)$ \\
\hline Ablation & $13(10.7 \%)$ \\
\hline Both & $7(5.8 \%)$ \\
\hline Salvage liver transplantation & $9(7.4 \%)$ \\
\hline Incidental tumors & $13(10.7 \%)$ \\
\hline \multicolumn{2}{|l|}{ ntraoperative factors } \\
\hline \multicolumn{2}{|l|}{ Surgical technique } \\
\hline Piggyback & $101(83.5 \%)$ \\
\hline Conventional & $20(16.5 \%)$ \\
\hline Packed RBC transfusions (units) & $4(0-48)$ \\
\hline FFP transfusions (units) & $8(0-50)$ \\
\hline Total operative time (hours) & $7.0(3.8-12.0)$ \\
\hline Duration of hepatectomy (hours) & $3.0(1.5-5.1)$ \\
\hline Warm ischemia (minutes) & $68.5(25.0-195.0)$ \\
\hline
\end{tabular}

$U C S F$ University of California, San Francisco, $A F P$ - fetoprotein, $R B C$ red blood cell, FFP fresh frozen plasma

${ }^{\text {a }}$ Based on explant pathology

${ }^{b}$ Calculated as a sum of estimated volumes of each tumor $\left(4 / 3 \pi r^{3}\right)$
Table 2 Associations between serum AFP concentration and other continuous variables

\begin{tabular}{llll}
\hline Variable 1 & Variable 2 & $\begin{array}{l}\text { Correlation } \\
\text { coefficient }\end{array}$ & $p$ \\
\hline AFP & Recipient age & 0.046 & 0.627 \\
AFP & Model for end-stage & -0.037 & 0.691 \\
& $\quad$ liver disease score & & \\
AFP & Size of the largest tumor & 0.185 & 0.050 \\
AFP & No. of tumors & 0.022 & 0.816 \\
AFP & Total tumor volume & 0.159 & 0.100 \\
\hline
\end{tabular}

factors for HCC recurrence in univariate and bivariate analyses. Linearity of the associations between AFP and recurrence hazard was assessed in general additive models and presented as spline functions. Hazard ratios (HRs) were presented with $95 \%$ confidence intervals $(95 \% \mathrm{CI})$. The level of statistical significance was set at 0.05. All analyses were computed in SAS version 9.3 (SAS Institute, Cary, NC, USA) and S-Plus version 6.1 (TIBCO Software, Palo Alto, CA, USA).

\section{Results}

Baseline characteristics of the 121 patients included in the study cohort are shown in Table 1. Median follow-up for the entire study cohort was 30 months. There was a positive correlation between AFP and the size of the largest tumor that verged on significance $(p=0.050)$ (Table 2). Significantly higher AFP was also noted in patients of female sex $(p=0.009)$, with hepatitis $C$ virus-related cirrhosis $(p=0.010)$, without hepatitis B virus infection $(p=0.032)$, or with nonincidental tumors $(p=0.024)$ (Table 3). Although the associations between AFP and microvascular invasion $(p=0.252)$ or tumor differentiation $(p=0.775)$ did not reach the level of significance, the median AFP level was markedly higher for patients with microvascular invasion or poorly differentiated tumors.

AFP was a significant risk factor for tumor recurrence $(p<0.001)$ in the univariate analyses (Table 4). Among the remaining factors, the hazard of recurrence was significantly influenced by the size of the largest tumor $(p<0.001)$, number of tumors $(p=0.017)$, total tumor volume $(p=0.001)$, tumors beyond the Milan criteria ( $p=0.022)$ or the Up-to-7 criteria $(p=0.021)$, recipient age $(p=0.044)$, donor sex $(p=0.009)$, and conventional LT technique $(p=0.004)$. Controlled for each of the potential confounders considered in this study, AFP retained significance as a risk factor for posttransplant HCC recurrence in all of the bivariate models (Table 5). Tumors of higher diameter $(p=0.010)$, those beyond the Milan criteria $(p=0.044)$, and female sex of the donor 
Table 3 Associations between serum AFP concentration and categoric variables

\begin{tabular}{|c|c|c|c|}
\hline \multirow[t]{2}{*}{ Variable } & \multicolumn{3}{|c|}{ AFP (ng/ml) } \\
\hline & Median & Range & $p$ \\
\hline Recipient sex & & & 0.009 \\
\hline Female & 124.3 & $3.3-17500.0$ & \\
\hline Male & 15.5 & $1.4-36208.0$ & \\
\hline Child-Turcotte-Pugh class & & & 0.173 \\
\hline A & 30.7 & $1.6-36208.0$ & \\
\hline $\mathrm{B}$ & 34.5 & $2.0-7053.0$ & \\
\hline $\mathrm{C}$ & 9.2 & $1.4-17500.0$ & \\
\hline Hepatitis $\mathrm{C}$ virus infection & & & 0.010 \\
\hline No & 10.6 & $1.9-7053.0$ & \\
\hline Yes & 43.1 & $1.4-36208.0$ & \\
\hline Hepatitis B virus infection & & & 0.032 \\
\hline No & 41.8 & $1.4-36208.0$ & \\
\hline Yes & 10.2 & $1.9-23231.0$ & \\
\hline Alcoholic cirrhosis & & & 0.358 \\
\hline No & 28.2 & $1.6-36208.0$ & \\
\hline Yes & 16.0 & $1.4-7053.0$ & \\
\hline Milan criteria & & & 0.196 \\
\hline Within & 18.8 & $1.6-813.3$ & \\
\hline Beyond & 39.5 & $1.4-36208.0$ & \\
\hline UCSF criteria & & & 0.648 \\
\hline Within & 25.2 & $1.6-23231.0$ & \\
\hline Beyond & 21.4 & $1.4-36208.0$ & \\
\hline Up-to-7 criteria & & & 0.500 \\
\hline Within & 24.7 & $1.6-23231.0$ & \\
\hline Beyond & 21.6 & $1.4-36208.0$ & \\
\hline Microvascular invasion & & & 0.252 \\
\hline No & 15.5 & $1.6-23231.0$ & \\
\hline Yes & 39.5 & $1.4-36208.0$ & \\
\hline Tumor differentiation & & & 0.775 \\
\hline Well & 41.8 & $2.6-7053.0$ & \\
\hline Moderate & 22.4 & $1.4-36208.0$ & \\
\hline Poor & 93.6 & $2.9-23231.0$ & \\
\hline Bilateral tumors & & & 0.698 \\
\hline No & 18.8 & $1.6-36208.0$ & \\
\hline Yes & 38.0 & $1.4-17500.0$ & \\
\hline Neoadjuvant therapy & & & 0.652 \\
\hline No & 21.1 & $1.4-23231.0$ & \\
\hline Yes & 31.6 & $1.6-36208.0$ & \\
\hline Salvage liver transplantation & & & 0.293 \\
\hline No & 20.6 & $1.4-23231.0$ & \\
\hline Yes & 141.0 & $1.9-36208.0$ & \\
\hline Incidental tumors & & & 0.024 \\
\hline No & 27.9 & $1.4-36208.0$ & \\
\hline Yes & 8.3 & $3.3-69.2$ & \\
\hline
\end{tabular}

( $p=0.029$ ) increased the risk of HCC recurrence independently of the pretransplant AFP level. All associations
Table 4 Univariates analyses of risk factors for recurrence during the first three posttransplant years

\begin{tabular}{llll}
\hline Factors & HR & $95 \%$ CI & $p$ \\
\hline Serum AFP concentration & $1.40^{\mathrm{a}}$ & $1.20-1.63$ & $<0.001$ \\
Size of the largest tumor & $1.48^{\mathrm{b}}$ & $1.20-1.83$ & $<0.001$ \\
No. of tumors & $1.21^{\mathrm{c}}$ & $1.03-1.41$ & 0.017 \\
Total tumor volume & $1.03^{\mathrm{d}}$ & $1.01-1.05$ & 0.001 \\
Microvascular invasion & 1.95 & $0.63-6.04$ & 0.248 \\
Poor tumor differentiation & 2.63 & $0.54-12.71$ & 0.230 \\
Bilateral tumors & 0.70 & $0.19-2.54$ & 0.586 \\
Neoadjuvant therapy & 1.50 & $0.49-4.62$ & 0.479 \\
Salvage liver transplantation & 2.69 & $0.59-12.20$ & 0.200 \\
Incidental tumors & 0.70 & $0.09-5.40$ & 0.734 \\
Tumors beyond Milan criteria & 4.55 & $1.25-16.67$ & 0.022 \\
Tumors beyond UCSF criteria & 2.54 & $0.85-7.58$ & 0.095 \\
Tumors beyond Up-to-7 criteria & 3.64 & $1.22-10.87$ & 0.021 \\
Recipient age & $0.62^{\mathrm{e}}$ & $0.39-0.99$ & 0.044 \\
Recipient sex & $0.76^{\mathrm{f}}$ & $0.23-2.45$ & 0.641 \\
Child-Turcotte-Pugh class C & 1.10 & $0.21-5.67$ & 0.913 \\
Model for end-stage liver disease & $1.03^{\mathrm{g}}$ & $0.94-1.12$ & 0.561 \\
Hepatitis C virus infection & 0.95 & $0.31-2.91$ & 0.931 \\
Hepatitis B virus infection & 1.39 & $0.47-4.14$ & 0.555 \\
Alcoholic cirrhosis & 1.01 & $0.22-4.56$ & 0.996 \\
Donor age & $0.76^{\mathrm{e}}$ & $0.50-1.16$ & 0.202 \\
Donor sex & $0.23^{\mathrm{f}}$ & $0.08-0.69$ & 0.009 \\
Cold ischemia & $1.45^{\mathrm{h}}$ & $0.84-2.52$ & 0.187 \\
Conventional technique for liver & 4.99 & $1.68-14.87$ & 0.004 \\
transplantation & & & \\
Packed RBC transfusions & $0.95^{\mathrm{i}}$ & $0.78-1.15$ & 0.569 \\
FFP transfusions & $0.86^{\mathrm{i}}$ & $0.66-1.11$ & 0.236 \\
Total operative time & $1.16^{\mathrm{h}}$ & $0.75-1.80$ & 0.503 \\
Duration of hepatectomy & $0.63^{\mathrm{h}}$ & $0.247-1.62$ & 0.340 \\
Warm ischemia & $1.05^{\mathrm{j}}$ & $0.85-1.29$ & 0.662 \\
\hline & & & \\
\hline & & &
\end{tabular}

$H R$ hazard ratio, $C I$ confidence interval

a increase by $100 \mathrm{ng} / \mathrm{ml}$

${ }^{b}$ Increase by $1 \mathrm{~cm}$

c Increase by 1 tumor

d Increase by $10 \mathrm{~cm}^{3}$

e Increase by 10 years

${ }^{\mathrm{f}}$ Male sex

$\mathrm{g}$ Increase by 1 point

${ }^{\mathrm{h}}$ Increase by $1 \mathrm{~h}$

${ }^{\mathrm{i}}$ Increase by 1 unit

j Increase by $10 \mathrm{~min}$

between AFP and posttransplant recurrence risk were nearly linear in the univariate analyses (linear effect $p<0.001$; nonlinear effect $p=0.434$ ) and bivariate analyses (linear effects: $p<0.001-0.034$; nonlinear effects $p=0.120-0.730$ ) (Fig. 1). 
Table 5 Association between AFP and the risk of tumor recurrence 3 years after exclusion of the impact of potential confounders in a series of bivariate models
Each row represents results of separate bivariate analysis

$H R$ hazard ratio, $L T$ liver transplantation

${ }^{\text {a }}$ Increase by $100 \mathrm{ng} / \mathrm{ml}$

b Increase by $1 \mathrm{~cm}$

c Increase by 1 tumor

d Increase by $10 \mathrm{~cm}^{3}$

e Tumors beyond

${ }^{f}$ Increase by 10 years

g Class $\mathrm{C}$

${ }^{\mathrm{h}}$ Increase by one point

${ }^{\mathrm{i}}$ Increase by $1 \mathrm{~h}$

${ }^{j}$ Increase by 1 unit

${ }^{\mathrm{k}}$ Increase by $10 \mathrm{~min}$

\begin{tabular}{|c|c|c|c|c|c|}
\hline Factor 1 & $\operatorname{HR}(95 \% \mathrm{CI})^{\mathrm{a}}$ & $p$ & Factor 2 & $\mathrm{HR}(95 \% \mathrm{CI})$ & $p$ \\
\hline AFP & $1.21(1.02-1.45)$ & 0.032 & Size of largest tumor & $1.41(1.09-1.82)^{b}$ & 0.010 \\
\hline AFP & $1.34(1.13-1.59)$ & $<0.001$ & No. of tumors & $1.10(0.92-1.31)^{c}$ & 0.314 \\
\hline AFP & $1.46(1.17-1.82)$ & $<0.001$ & Total tumor volume & $1.02(0.99-1.04)^{\mathrm{d}}$ & 0.112 \\
\hline AFP & $1.43(1.20-1.71)$ & $<0.001$ & Microvascular invasion & $1.86(0.51-6.82)$ & 0.348 \\
\hline AFP & $1.43(1.22-1.69)$ & $<0.001$ & Poor tumor differentiation & $1.92(0.39-9.44)$ & 0.421 \\
\hline AFP & $1.42(1.21-1.66)$ & $<0.001$ & Bilateral tumors & $0.62(0.16-2.44)$ & 0.489 \\
\hline AFP & $1.40(1.20-1.63)$ & $<0.001$ & Neoadjuvant therapy & $1.49(0.44-5.01)$ & 0.524 \\
\hline AFP & $1.40(1.20-1.63)$ & $<0.001$ & Salvage LT & $1.56(0.19-12.78)$ & 0.681 \\
\hline AFP & $1.38(1.19-1.61)$ & $<0.001$ & Incidental tumors & - & 0.995 \\
\hline AFP & $1.31(1.14-1.52)$ & $<0.001$ & Milan criteria & $8.62(1.06-71.43)^{\mathrm{e}}$ & 0.044 \\
\hline AFP & $1.36(1.16-1.58)$ & $<0.001$ & UCSF criteria & $2.34(0.65-8.47)^{\mathrm{e}}$ & 0.196 \\
\hline AFP & $1.33(1.14-1.54)$ & $<0.001$ & Up-to-7 criteria & $3.30(0.89-12.20)^{\mathrm{e}}$ & 0.074 \\
\hline AFP & $1.39(1.19-1.62)$ & $<0.001$ & Recipient age & $0.82(0.43-1.57)^{\mathrm{f}}$ & 0.555 \\
\hline AFP & $1.49(1.26-1.76)$ & $<0.001$ & Recipient sex (male) & $3.10(0.70-13.81)$ & 0.137 \\
\hline AFP & $1.47(1.24-1.75)$ & $<0.001$ & Child-Turcotte-Pugh class & $1.03(0.31-3.41)^{\mathrm{g}}$ & 0.967 \\
\hline AFP & $1.39(1.19-1.62)$ & $<0.001$ & Model for end-stage liver disease & $1.03(0.91-1.16)^{\mathrm{h}}$ & 0.647 \\
\hline AFP & $1.40(1.21-1.63)$ & $<0.001$ & Hepatitis $\mathrm{C}$ virus infection & $0.84(0.24-2.90)$ & 0.781 \\
\hline AFP & $1.40(1.20-1.64)$ & $<0.001$ & Hepatitis B virus infection & $1.01(0.29-3.47)$ & 0.986 \\
\hline AFP & $1.40(1.20-1.63)$ & $<0.001$ & Alcoholic liver disease & $1.35(0.28-6.44)$ & 0.710 \\
\hline AFP & $1.38(1.16-1.65)$ & $<0.001$ & Donor age & $1.07(0.66-1.74)^{\mathrm{f}}$ & 0.791 \\
\hline AFP & $1.36(1.16-1.59)$ & $<0.001$ & Donor sex (male) & $0.24(0.07-0.86)$ & 0.029 \\
\hline AFP & $1.31(1.08-1.60)$ & 0.008 & Cold ischemia & $1.43(0.81-2.54)^{\mathrm{i}}$ & 0.221 \\
\hline AFP & $1.34(1.14-1.57)$ & $<0.001$ & Conventional LT technique & $2.52(0.68-9.34)$ & 0.166 \\
\hline AFP & $1.33(1.12-1.58)$ & 0.001 & Packed RBC transfusions & $0.93(0.74-1.16)^{\mathrm{j}}$ & 0.501 \\
\hline AFP & $1.37(1.14-1.64)$ & $<0.001$ & FFP transfusions & $0.84(0.66-1.06)^{\mathrm{j}}$ & 0.141 \\
\hline AFP & $1.33(1.10-1.60)$ & 0.003 & Total operative time & $0.97(0.63-1.48)^{\mathrm{i}}$ & 0.883 \\
\hline AFP & $1.37(1.10-1.70)$ & 0.005 & Duration of hepatectomy & $0.55(0.21-1.44)^{\mathrm{i}}$ & 0.226 \\
\hline AFP & $1.40(1.14-1.72)$ & 0.001 & Warm ischemia & $0.96(0.79-1.17)^{\mathrm{k}}$ & 0.696 \\
\hline
\end{tabular}

exhibited superior recurrence-free survival at 5 years (100\%) compared to patients within these criteria but with AFP $>100 \mathrm{ng} / \mathrm{ml})(69.0 \%)$ or beyond these criteria and AFP $<200 \mathrm{ng} / \mathrm{ml}$ (64.3\%; $p=0.005)$ (Fig. 3b). Similarly, the 5-year recurrence-free survival of patients within the Up-to-7 criteria and AFP $<100 \mathrm{ng} / \mathrm{ml}(100 \%)$ was superior to the corresponding rates in patients within and beyond these criteria and AFP $>100 \mathrm{ng} / \mathrm{ml}(71.9 \%)$ or $<100 \mathrm{ng} / \mathrm{ml}(47.8 \%)$, respectively $(p=0.001)$ (Fig. 3c).

\section{Discussion}

The Milan criteria are consistently being criticized for being too restrictive $[7,8,21,22]$. In fact, it has been shown in several studies that the use of expanded criteria, such as the UCSF or Up-to-7 proposals, might provide results comparable to those provided by the use of Milan criteria $[7,8,23,24]$. At the same time, large-scale national-level studies indicate that HCC is associated with inferior posttransplant outcomes compared to other 


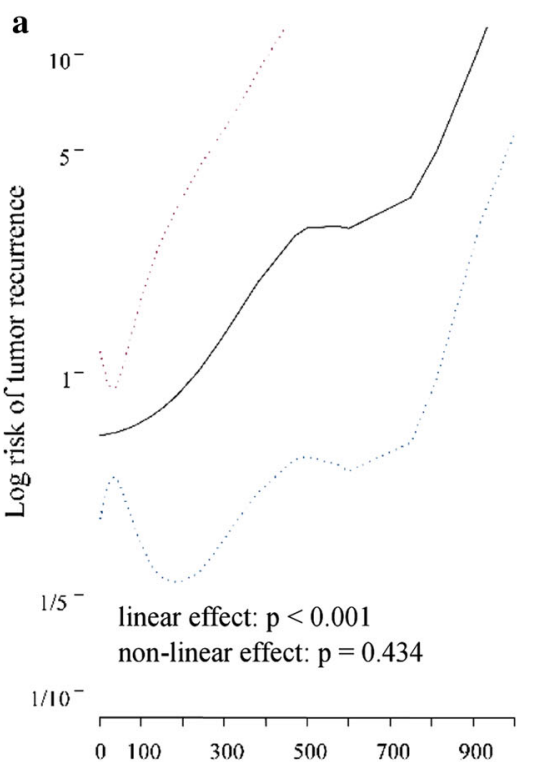

Serum alpha-fetoprotein concentration ( $\mathrm{ng} / \mathrm{mL}$ )

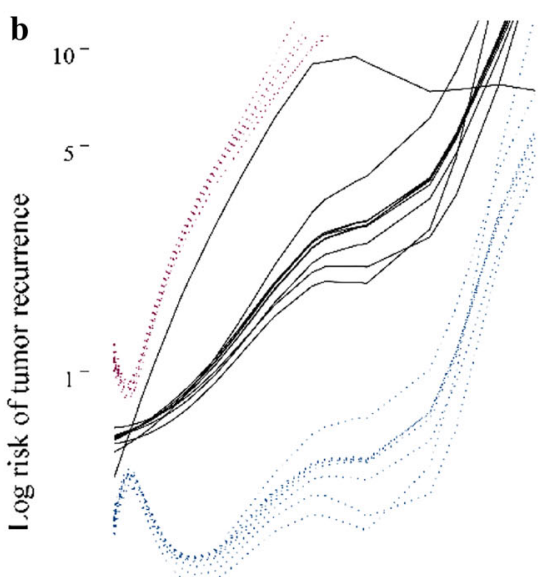

$1 / 5^{-} \quad$ linear effects: $p<0.001-0.032$ non-linear effects: p $0.120-0.600$

$1 / 10^{-}$

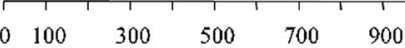

Scrum alpha-fetoprotcin concentration $(\mathrm{ng} / \mathrm{mL})$

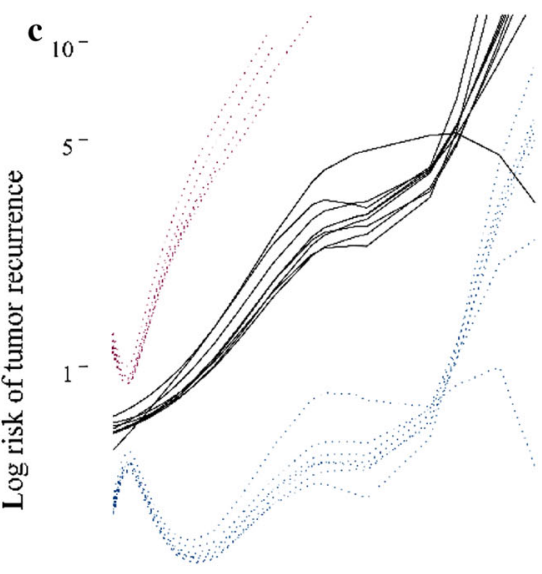

$1 / 5^{-} \quad$ linear effects: $p<0.001-0.034$ non-linear effects: $\mathrm{p} 0.230-0.700$

$1 / 10^{-}$

$\begin{array}{llllll}0 & 100 & 300 & 500 & 700 & 900\end{array}$

Scrum alpha-fetoprotein concentration $(\mathrm{ng} / \mathrm{mL})$

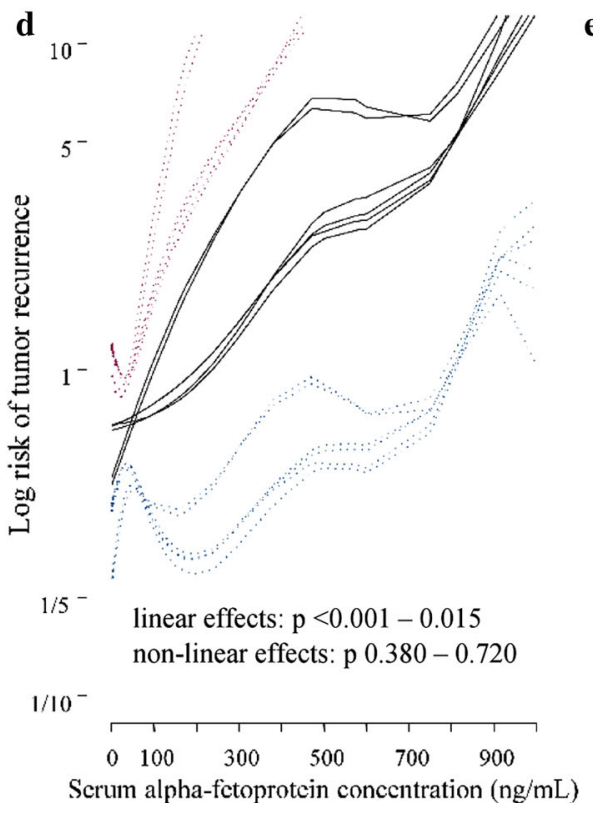

${ }_{10}$

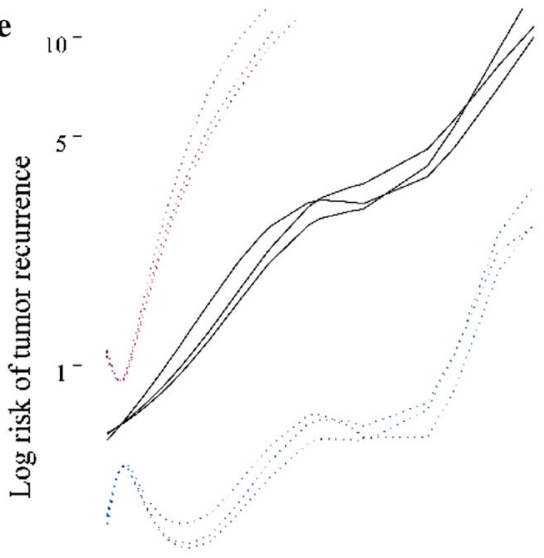

$1 / 5^{-} \quad$ linear effects: $p<0.001$

non-linear effects: p $0.650-0.730$

$1 / 10^{-}$

$\begin{array}{llllll}0 & 100 & 300 & 500 & 700 & 900\end{array}$

Serum alpha-fetoprotein concentration $(\mathrm{ng} / \mathrm{mL})$
Fig. 1 Associations between serum $\alpha$-fetoprotein (AFP) concentration and the risk of posttransplant tumor recurrence in the univariate analysis (a) and adjusted for the impact of potential confounders,

indications $[9,10]$. In fact, the "Metroticket" concept of the authors of Up-to-7 criteria illustrates that expansion of the limits for morphologic tumor features during selection to undergo LT would further alter the posttransplant outcomes [8]. Moreover, the number of LTs performed for HCC has rapidly increased over the last several years [10]. With the limited pool of deceased donors, this situation might be harmful to patients with other indications for LT because it prolongs their waiting time, resulting in increased deaths while still on the waiting list. Under these including general factors (b), tumor-related factors (c), intraoperative factors (d), and fulfillment of selection criteria (e). Risk curves (solid lines) are presented with $95 \%$ confidence intervals (dotted lines)

circumstances, the HCC Consensus Group stated that the outcomes after LT for HCC and other indications should be comparable [11]. Thus, the optimal selection criteria should ideally bring the risk of posttransplant tumor recurrence to zero.

The results of this study that support the rationale for including AFP in the existing criteria are in line with the findings in previous reports. Notably, both AFP and the size of the largest tumor were independent predictors of tumor recurrence, whereas the number of tumors did not 
Table 6 Associations between serum AFP concentration and risk of posttransplant tumor recurrence at 3 years in patients within and beyond the Milan, University of California, San Francisco, and Up-to7 criteria

\begin{tabular}{lllr}
\hline Criteria & Factor & HR $(95 \% \text { CI })^{\mathrm{a}}$ & $p$ \\
\hline Milan & & $1.09(0.47-2.53)$ & 0.834 \\
Within & AFP & $1.32(1.14-1.53)$ & $<0.001$ \\
Beyond & AFP & & \\
UCSF & & $1.64(1.21-2.21)$ & 0.001 \\
Within & AFP & $1.24(1.04-1.47)$ & 0.015 \\
Beyond & AFP & & \\
Up-to-7 & & $1.66(1.22-2.25)$ & 0.001 \\
Within & AFP & $1.21(1.03-1.43)$ & 0.023 \\
Beyond & AFP & & \\
\hline
\end{tabular}

${ }^{a}$ Per $100 \mathrm{ng} / \mathrm{ml}$ increase

reach the level of significance after adjusting for the impact of the AFP level. However, as the nature of this study was retrospective, such observation clearly does not preclude the importance of the number of tumors during the qualification of patients with HCC for transplantation due to the potential of selection bias. Accordingly, the existing and already popular selection criteria were used to determine the optimal AFP cutoffs subsequently used to develop a strategy for their modification. Analysis of the spline curves revealed linearity of the associations between AFP and the risk of $\mathrm{HCC}$ recurrence. Hence, these cutoffs were based on prediction curves derived from Kaplan-Meier recurrence-free survival estimates.

The major finding of the present study is that the estimated rate of tumor recurrence at 5 years was $0 \%$ (corresponding to recurrence-free survival of $100 \%$ ) in patients with AFP levels $<100 \mathrm{ng} / \mathrm{ml}$ and with a tumor burden within the limits of either the UCSF or Up-to-7 criteria. These results support selection of HCC patients with such characteristics for LT either as an addition to the Milan criteria or even their replacement. In both cases, prospective validation of the UCSF or Up-to-7 criteria modified with the $100 \mathrm{ng} / \mathrm{ml}$ AFP cutoff in an unselected cohort of HCC patients would be necessary to confirm the rationale for their clinical use. Nevertheless, the proposal for slight expansion of the Milan criteria only to patients with minimal risk of HCC tumor recurrence seems to be a
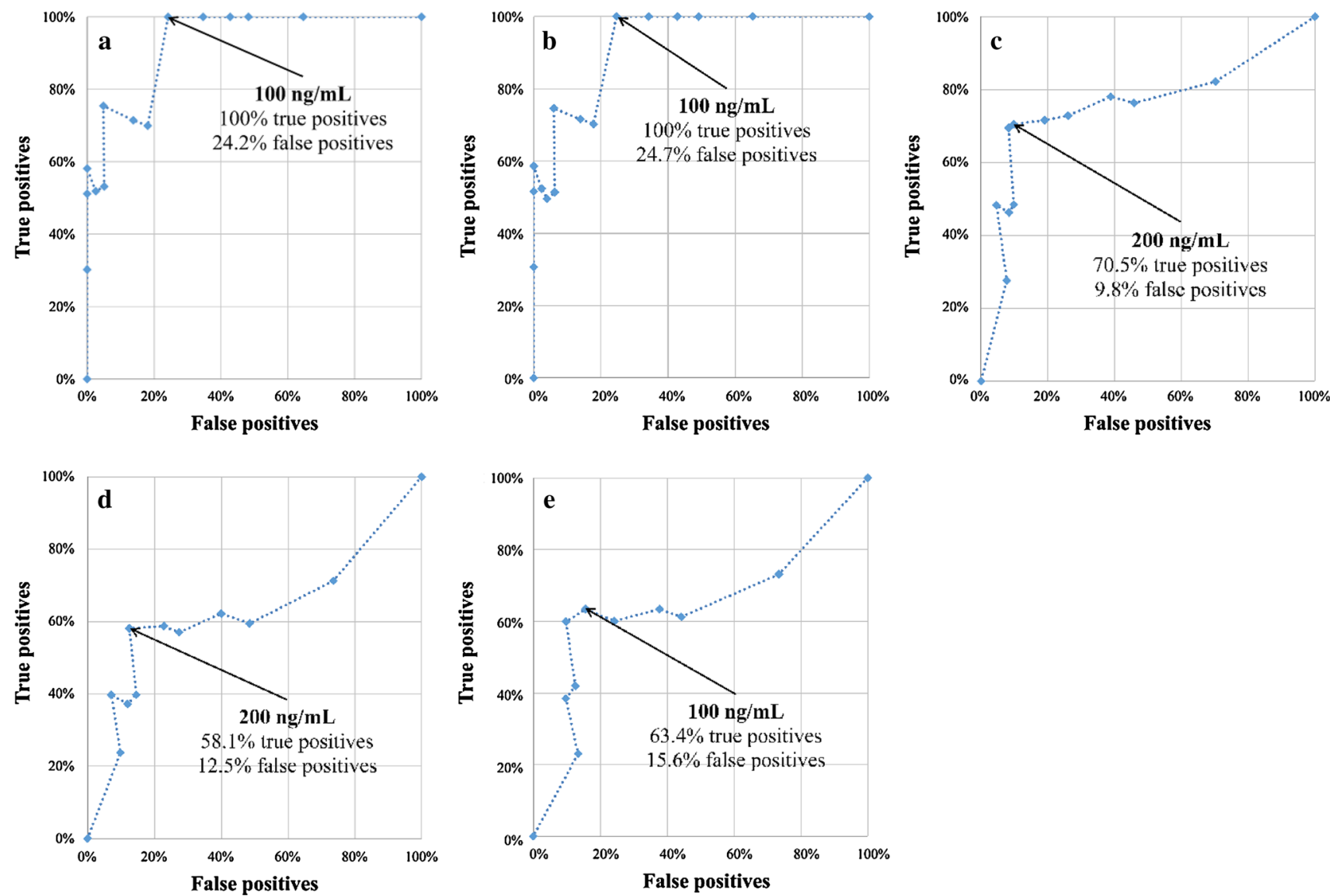

Fig. 2 Estimation of the optimal cutoffs for serum AFP concentration for predicting posttransplant tumor recurrence in patients within the University of California, San Francisco (UCSF) (a) and Up-to-7 (b) criteria and beyond the Milan (c), UCSF (d), and Up-to-7 (e) criteria 

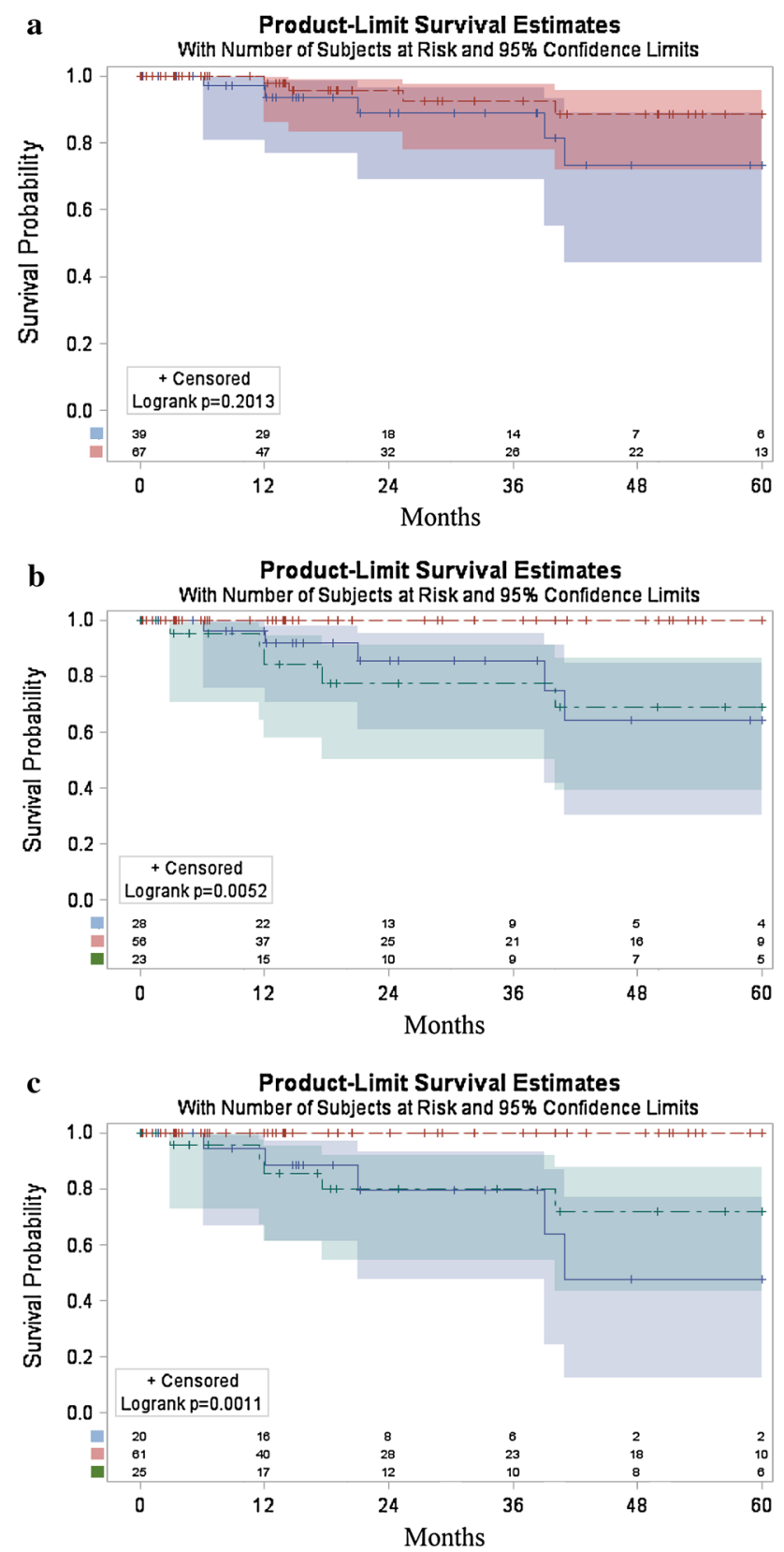

Fig. 3 Recurrence-free survivals. a Patients within the Milan criteria (dashed line) and beyond the Milan criteria (solid line) with AFP $<200 \mathrm{ng} / \mathrm{ml}$. b Patients within UCSF criteria with AFP $<100 \mathrm{ng} / \mathrm{ml}$ (dashed line) and $>100 \mathrm{ng} / \mathrm{ml}$ (dashed-dotted line) and beyond the UCSF criteria with AFP $<200 \mathrm{ng} / \mathrm{ml}$ (solid line). c Patients within Up-to-7 criteria with AFP $<100 \mathrm{ng} / \mathrm{ml}$ (dashed line) and $>100 \mathrm{ng} / \mathrm{ml}$ (dashed-dotted line) and beyond Up-to-7 criteria (solid line) with AFP $<100 \mathrm{ng} / \mathrm{ml}$. Survival curves are presented with $95 \%$ confidence intervals

reasonable alternative to expanding the selection criteria to include patients with moderate risk that is comparable to or slightly higher than that provided by the Milan criteria.

Interestingly, recurrence-free survival of patients within the UCSF criteria and AFP levels over the estimated cutoff was similar to those beyond them but with AFP levels
$<200 \mathrm{ng} / \mathrm{ml}$. In contrast, patients beyond the Up-to-7 criteria and AFP $<100 \mathrm{ng} / \mathrm{ml}$ exhibited a markedly higher recurrence rate than patients within the Up-to-7 criteria but with AFP $>100 \mathrm{ng} / \mathrm{ml}$. Most importantly, tumor recurrences were observed in a remarkable proportion of patients from each of these subgroups. Considering the principle of minimizing the risk of tumor recurrence to achieve outcomes comparable to those seen with other indications, selection of these patients for LT is highly controversial.

Several selection criteria utilizing AFP have been proposed to date, such as the Hangzhou criteria, Seoul criteria, and total tumor volume/AFP (TTV/AFP) criteria. Seoul criteria were defined as the size of the largest tumor $\leq 5 \mathrm{~cm}$ and AFP $<400 \mathrm{ng} / \mathrm{ml}$ regardless of the number of tumors [25]. The authors of the Hangzhou criteria proposed an identical cutoff. They stated that all patients who had well or moderately differentiated tumors and AFP $<400 \mathrm{ng} / \mathrm{ml}$ might be considered eligible for LT [26]. However, according to the present study, a cutoff for AFP of $400 \mathrm{ng} / \mathrm{ml}$ would lead to a marked increase in the recurrence rate, even in patients who were within the UCSF or Up-to-7 criteria, which are clearly were more restrictive with respect to morphological features than the Seoul or Hangzhou proposals. Conversely, TTV/AFP criteria (TTV $<115 \mathrm{~cm}^{3}+$ AFP $<400 \mathrm{ng} / \mathrm{ml}$ ) were associated with superior survival rates in the original study [27] and very low recurrence rate at 5 years in their recent retrospective validation study at the same institution as the authors of the current study [28]. However, as both studies were retrospective and the morphologic limits quite liberal, the results might have been subject to selection bias. Moreover, their fulfillment still did not eliminate the problem of HCC recurrence.

Duvoux et al. [29] also evaluated the concept of combining AFP with morphologic features of the tumor. Based on the AFP levels, tumor size, and number of tumor nodules, the authors of that highly relevant report introduced a predictive model for tumor recurrence that is superior to the Milan criteria for categorizing patients into low- and high-risk groups. In contrast to this interesting proposal of absolute replacement of the Milan criteria with a new risk index, the results of the present study point toward the potential of slight expansion of the former without a negative impact on the risk of recurrence.

In a study based on 92 patients after LT for HCC, Yaprak et al. [30] found that those at low- and high-risk of tumor recurrence can be distinguished based on their AFP level. However, there are several differences between their study and the present study. First, the cutoffs for AFP utilized by Yaprak et al. were arbitrary and hence identical for patients within and beyond the Milan criteria. Notably, the results of the present study indicate that the optimal cutoffs may substantially differ between patients within and beyond 
particular selection criteria, which seems natural given the correlation between AFP and tumor size. Moreover, the results obtained in the present study highlight the linear nature of the association between AFP and the risk of recurrence, which may prove useful in clinical practice regardless of whether the suggested expansion of selection criteria is adopted. Finally, the results not only confirm the prognostic role of AFP in LT for HCC. They provide additional data to support the rationale for safe utilization of the expanded criteria when limited by the AFP level.

Finally, some authors have suggested that a pretransplant AFP slope is superior to single absolute values [31]. According to a study by Dumitra et al. [32] based on the data of 92 HCC liver transplant recipients, a rising AFP slope was strongly associated with tumor recurrence. However, the positive predictive value of the rising AFP slope was only $25.0 \%$ in that study. On the other hand, results of a large study including 6,817 patients by Merani et al. [33] indicated that the last preoperative AFP level is what matters the most, as patients with stable high AFP and those with originally low but increasing AFP have similar prognoses.

Notably, the usefulness of AFP as an important risk factor for postoperative outcomes was also previously confirmed for patients with HCCs in a noncirrhotic setting. Specifically, Witjes et al. [34] analyzed the data from 94 patients with HCC in noncirrhotic livers and found that AFP and the presence of microvascular invasion were the only independent risk factors for tumor recurrence. Although such patients are not currently considered suitable for LT, these results indicate that AFP should also be taken into consideration when establishing selection criteria for LT in future studies.

Among other potential markers for HCC aggressiveness in a liver transplant setting, des- $\gamma$-carboxyprothrombin (DCP) is the one most frequently studied. The results of other studies comparing AFP and DCP as predictors of poor outcomes are contradictory. Hence, there is no clear evidence for superiority of one over another [35, 36]. Unfortunately, pretransplant DCP was not assessed in patients included in the present study.

\section{Conclusion}

Given the minimal risk of posttransplant recurrence, patients who are within Up-to-7 or UCSF criteria should be considered eligible for LT provided the AFP level is $<100 \mathrm{ng} / \mathrm{ml}$.

\section{Conflict of interest None.}

Open Access This article is distributed under the terms of the Creative Commons Attribution License which permits any use, distribution, and reproduction in any medium, provided the original author(s) and the source are credited.

\section{References}

1. Jemal A, Bray F, Center MM et al (2011) Global cancer statistics. CA Cancer J Clin 61:69-90

2. Barbara L, Benzi G, Gaiani S et al (1992) Natural history of small untreated hepatocellular carcinoma in cirrhosis: a multivariate analysis of prognostic factors of tumor growth rate and patient survival. Hepatology 16:132-137

3. Shen A, Tang C, Wang Y et al (2013) A systematic review of sorafenib in Child-Pugh A patients with unresectable hepatocellular carcinoma. J Clin Gastroenterol 47:871-880

4. Forner A, Llovet JM, Bruix J (2012) Hepatocellular carcinoma. Lancet 379:1245-1255

5. Ringe B, Pichlmayr R, Wittekind C et al (1991) Surgical treatment of hepatocellular carcinoma: experience with liver resection and transplantation in 198 patients. World J Surg 15:270-285

6. Mazzaferro V, Regalia E, Doci R et al (1996) Liver transplantation for the treatment of small hepatocellular carcinomas in patients with cirrhosis. N Engl J Med 334:693-699

7. Yao FY, Ferrell L, Bass NM et al (2001) Liver transplantation for hepatocellular carcinoma: expansion of the tumor size limits does not adversely impact survival. Hepatology 33:1394-1403

8. Mazzaferro V, Llovet JM, Miceli R et al (2009) Predicting survival after liver transplantation in patients with hepatocellular carcinoma beyond the Milan criteria: a retrospective, exploratory analysis. Lancet Oncol 10:35-43

9. Mailey B, Buchberg B, Prendergast C et al (2009) A diseasebased comparison of liver transplantation outcomes. Am Surg 75:901-908

10. Singal AK, Guturu P, Hmoud B et al (2013) Evolving frequency and outcomes of liver transplantation based on etiology of liver disease. Transplantation 95:755-760

11. Clavien PA, Lesurtel M, Bossuyt PM et al (2012) Recommendations for liver transplantation for hepatocellular carcinoma: an international consensus conference report. Lancet Oncol 13:e11e22

12. Bruix J, Sherman M (2011) American Association for the Study of Liver Diseases. Management of hepatocellular carcinoma: an update. Hepatology 53:1020-1022

13. European Association for Study of Liver; European Organisation for Research and Treatment of Cancer (2012) EASL-EORTC clinical practice guidelines: management of hepatocellular carcinoma. Eur J Cancer 48:599-641

14. Grąt M, Hołówko W, Grzegorczyk K et al (2011) Long-term results of liver resection in the treatment of patients with hepatocellular carcinoma. Pol Przegl Chir 83:319-324

15. Grąt M, Kornasiewicz O, Lewandowski Z et al (2013) The impact of surgical technique on the results of liver transplantation in patients with hepatocellular carcinoma. Ann Transplant 18:448-459

16. Graham JA, Melancon JK, Shetty K et al (2013) Liver transplantation should be offered to patients with small solitary hepatocellular carcinoma and a positive serum alpha fetoprotein rather than resection. Am J Surg 205:374-380

17. Lee YH, Hsia CY, Hsu CY et al (2013) Total tumor volume is a better marker of tumor burden in hepatocellular carcinoma defined by the Milan criteria. World J Surg 37:1348-1355

18. Eguchi S, Takatsuki M, Hidaka M et al (2010) Predictor for histological microvascular invasion of hepatocellular carcinoma: a lesson from 229 consecutive cases of curative liver resection. World J Surg 34:1034-1038 
19. Krawczyk M, Grąt M, Barski K et al (2012) 1000 liver transplantations at the Department of General, Transplant and Liver Surgery, Medical University of Warsaw-analysis of indications and results. Pol Przegl Chir 84:304-312

20. Kaido T, Ogawa K, Mori A et al (2013) Usefulness of the Kyoto criteria as expanded selection criteria for liver transplantation for hepatocellular carcinoma. Surgery 154:1053-1060

21. Fan J, Yang GS, Fu ZR et al (2009) Liver transplantation outcomes in 1,078 hepatocellular carcinoma patients: a multi-center experience in Shanghai, China. J Cancer Res Clin Oncol 135:1403-1412

22. Herrero JI, Sangro B, Pardo F et al (2008) Liver transplantation in patients with hepatocellular carcinoma across Milan criteria. Liver Transplant 14:272-278

23. Lei J, Yan L (2012) Comparison between living donor liver transplantation recipients who met the Milan and UCSF criteria after successful downstaging therapies. J Gastrointest Surg $16: 2120-2125$

24. de Ataide EC, Garcia M, Mattosinho TJ et al (2012) Predicting survival after liver transplantation using up-to-seven criteria in patients with hepatocellular carcinoma. Transplant Proc 44:2438-2440

25. Kwon CH, Kim DJ, Han YS et al (2007) HCC in living donor liver transplantation: can we expand the Milan criteria? Dig Dis 25:313-319

26. Zheng SS, Xu X, Wu J et al (2008) Liver transplantation for hepatocellular carcinoma: Hangzhou experiences. Transplantation 85:1726-1732

27. Toso C, Asthana S, Bigam DL et al (2009) Reassessing selection criteria prior to liver transplantation for hepatocellular carcinoma utilizing the scientific registry of transplant recipients database. Hepatology 49:832-838

28. Grat M, Kornasiewicz O, Hołówko W et al (2013) Evaluation of total tumor volume and pretransplantation $\alpha$-fetoprotein level as selection criteria for liver transplantation in patients with hepatocellular cancer. Transplant Proc 45:1899-1903

29. Duvoux C, Roudot-Thoraval F, Decaens T et al (2012) Liver transplantation for hepatocellular carcinoma: a model including $\alpha$-fetoprotein improves the performance of Milan criteria. Gastroenterology 143:986-994

30. Yaprak O, Akyildiz M, Dayangac M et al (2012) AFP level and histologic differentiation predict the survival of patients with liver transplantation for hepatocellular carcinoma. Hepatobiliary Pancreat Dis Int 11:256-261

31. Han K, Tzimas GN, Barkun JS et al (2007) Preoperative alphafetoprotein slope is predictive of hepatocellular carcinoma recurrence after liver transplantation. Can J Gastroenterol 21:39-45

32. Dumitra TC, Dumitra S, Metrakos PP et al (2013) Pretransplantation $\alpha$-fetoprotein slope and Milan criteria: strong predictors of hepatocellular carcinoma recurrence after transplantation. Transplantation 95:228-233

33. Merani S, Majno P, Kneteman NM et al (2011) The impact of waiting list alpha-fetoprotein changes on the outcome of liver transplant for hepatocellular carcinoma. J Hepatol 55:814-819

34. Witjes CD, Polak WG, Verhoef $C$ et al (2012) Increased alphafetoprotein serum level is predictive for survival and recurrence of hepatocellular carcinoma in non-cirrhotic livers. Dig Surg 29:522-528

35. Fujiki M, Takada Y, Ogura Y et al (2009) Significance of desgamma-carboxy prothrombin in selection criteria for living donor liver transplantation for hepatocellular carcinoma. Am J Transplant 9:2362-2371

36. Shindoh J, Sugawara Y, Nagata R et al (2014) Evaluation methods for pretransplant oncologic markers and their prognostic impacts in patient undergoing living donor liver transplantation for hepatocellular carcinoma. Transpl Int 27:391-398 